\title{
NEW RESULTS FOR OSCILLATORY PROPERTIES OF NEUTRAL DIFFERENTIAL EQUATIONS WITH A $p$-LAPLACIAN LIKE OPERATOR
}

\author{
O. BAZIGHIFAN, S. R. GRACE, J. ALZABUT, AND A. ÖZBEKLER
}

Received 26 April, 2020

\begin{abstract}
Results reported in this paper provide a generalization for some previously obtained results. Based on comparing with the oscillatory behavior of first-order delay equations, we provide new oscillation criteria for the solutions of even-order neutral differential equations with a $p$-Laplacian like operator. The proposed theorems not only provide totally different approach but also essentially improve a number of results reported in the literature. To demonstrate the advantage of our results, we present two examples.
\end{abstract}

2010 Mathematics Subject Classification: 34C10; 34K11

Keywords: even-order differential equations, neutral delay, oscillation, $p$-Laplacian operator

\section{INTRODUCTION}

Recently, it has been recognized that higher order neutral differential equations can describe many real life applications; see $[1,13]$. As a result of this, the qualitative behavior of solutions for these equations have been the object of many scholars during the previous years $[3,7,10-12,14,15,18,22,23]$. Particular emphasis has been given to the study of oscillatory behavior of these equations which have been under investigation by using different methods and various techniques; we refer to the papers $[4-6,9,17,19-21]$. The consideration of higher-order equations was motivated by the attempt to promote the work and obtain a general platform that covers all particular cases. The consideration of equations incorporating the $p$-Laplacian operator has been one way to generalize existing result in the literature $[2,16,26]$.

The present paper deals with the investigation of the qualitative behavior of even order neutral differential equation

$$
\left(b(t) \Phi_{p}\left[w^{(\kappa-1)}(t)\right]\right)^{\prime}+q(t) \Phi_{p}[y(\delta(t))]=0 ; \quad t \geq t_{0}, \quad(\kappa=4,6,8, \ldots)
$$

where $\Phi_{p}[s]=|s|^{p-2} s, p>1$ and

$$
w(t):=y(t)+a(t) y(\tau(t)) .
$$

The main results are obtained under the following conditions: 
(i) $b(t)$ is a positive continuous function on $\left[t_{0}, \infty\right)$ with that $b^{\prime}(t) \geq 0$ and

$$
\int_{t_{0}}^{\infty}[b(s)]^{-1 /(p-1)} \mathrm{d} s=\infty .
$$

(ii) $a(t)$ and $q(t)$ are continuous functions on $\left[t_{0}, \infty\right)$ with $q(t)>0,0 \leq a(t)<$ $a_{0}<\infty$, and that $q(t) \not \equiv 0$ for large values of $t$.

(iii) $\tau \in C^{1}\left[t_{0}, \infty\right), \delta \in C\left[t_{0}, \infty\right), \tau^{\prime}(t)>0, \tau(t) \leq t$ and that

$$
\lim _{t \rightarrow \infty} \tau(t)=\lim _{t \rightarrow \infty} \delta(t)=\infty \text {. }
$$

By establishing a new oscillation theorem that compares the higher-order equation (1.1) with a couple of first-order delay differential equations whose oscillatory behavior is known, we improve some existing results in the literature. Examples are presented to illustrate the advantage of our results over previously obtained theorems.

For the sake of comparison, we review some previous results. In [25], Zafer proved that the even-order differential equation

$$
w^{(\kappa)}(t)+q(t) y(\delta(t))=0
$$

is oscillatory if

$$
\liminf _{t \rightarrow \infty} \int_{\delta(t)}^{t} Q(s) \mathrm{d} s>\frac{1}{e}(\kappa-1) 2^{(\kappa-1)(\kappa-2)},
$$

where $Q(t):=\delta^{\kappa-1}(t) q(t)[1-a(\delta(t))]$. In a similar approach, Zhang and Yan [27] proved that (1.3) is oscillatory if

$$
\liminf _{t \rightarrow \infty} \int_{\delta(t)}^{t} Q(s) \mathrm{d} s>\frac{1}{e}(\kappa-1) ! .
$$

It is easy to see that $(\kappa-1) !<(\kappa-1) 2^{(\kappa-1)(\kappa-2)}$ for $\kappa>3$, and hence the results obtained in [27] improve those of Zafer [25].

For non-linear equation, Xing et al. [24] proved that Eq. (1.1) is oscillatory if

$$
\left(\delta^{-1}\right)^{\prime}(t) \geq \delta_{0}>0, \quad \tau^{\prime}(t) \geq \tau_{0}>0, \quad \tau^{-1}(\delta(t))<t
$$

and

$$
\liminf _{t \rightarrow \infty} \int_{\tau^{-1}(\delta(t))}^{t} \frac{\widehat{q}(s)}{b(s)} s^{\alpha(\kappa-1)} \mathrm{d} s>\frac{1}{e \delta_{0}}\left(1+\frac{a_{0}^{\alpha}}{\tau_{0}}\right)[(\kappa-1) !]^{\alpha},
$$

where $\widehat{q}(t):=\min \left\{q\left(\delta^{-1}(t)\right), q\left(\delta^{-1}(\tau(t))\right)\right\}$.

\section{HYPOTHESES AND PRELIMINARIES}

For our purpose, we define the following notations:

$$
a_{k}(t):=\frac{1}{a\left(\tau^{-1}(t)\right)}\left(1-\frac{\varepsilon\left[\tau^{-1}\left(\tau^{-1}(t)\right)\right]^{k-1}}{\left[\tau^{-1}(t)\right]^{k-1} a\left(\tau^{-1}\left(\tau^{-1}(t)\right)\right)}\right) ; \quad k=2, \ldots, \kappa,
$$




$$
R_{0}(t):=\left(\frac{1}{b(t)} \int_{t}^{\infty} q(s)\left[a_{2}(\delta(s))\right]^{\alpha} \mathrm{d} s\right)^{1 /(p-1)}
$$

and

$$
R_{m}(t):=\int_{t}^{\infty} R_{m-1}(s) \mathrm{d} s ; \quad m=1,2, \ldots, \kappa-3 .
$$

To complete the main results, we need the following lemmas.

Lemma 1. Let $y \in C^{n}\left(\left[t_{0}, \infty\right),(0, \infty)\right)$. Assume that $y^{(n)}(t)$ is of fixed sign and not identically zero on $\left[t_{0}, \infty\right)$, and that there exists a $t_{1} \geq t_{0}$ such that $y^{(n-1)}(t) y^{(n)}(t) \leq 0$ for all $t \geq t_{1}$. If

$$
\lim _{t \rightarrow \infty} y(t) \neq 0,
$$

then for every $\mu \in(0,1)$ there exists $t_{\mu} \geq t_{1}$ such that

$$
y(t) \geq \frac{\mu}{(n-1) !}\left|y^{(n-1)}(t)\right| t^{n-1}
$$

for all $t \geq t_{\mu}$.

Lemma 2. Assume that $f, g \geq 0$ and $\beta$ is a positive real number. Then the inequalities

$$
(f+g)^{\beta} \leq 2^{\beta-1}\left(f^{\beta}+g^{\beta}\right) ; \quad \beta \geq 1
$$

and

$$
(f+g)^{\beta} \leq f^{\beta}+g^{\beta} ; \quad \beta \leq 1
$$

hold.

Lemma 3. If the function y satisfies $y^{(i)}(t)>0, i=0,1, \ldots, n$, and $y^{(n+1)}(t)<0$, then we have

$$
n y(t) \geq \varepsilon t y^{\prime}(t)
$$

for $\varepsilon \in(0,1)$.

Lemma 4. Assume that $y$ is an eventually positive solution of Eq. (1.1). Then, there exist two possible cases:

(a) $w(t)>0, w^{\prime}(t)>0, w^{\prime \prime}(t)>0, \ldots, w^{(n-1)}(t)>0, w^{(n)}(t)<0$

(b) $w(t)>0, w^{(m)}(t)>0, w^{(m+1)}(t)<0$ for all odd integers $m \in\{1,3, \ldots, \kappa-3\}, w^{(\kappa-1)}(t)>0, w^{(\kappa)}(t)<0$,

for $t \in\left[t_{1}, \infty\right)$ for some $t_{1} \geq t_{0}$ sufficiently large.

The lemmas given above can be found in [1, Lemma 2.2.3], [3, Lemma 1, Lemma 2], [8] and [23, Lemma 1.2], respectively. 


\subsection{Oscillation Criteria}

The following is the main result.

Theorem 1. Let

$$
\frac{\left[\tau^{-1}\left(\tau^{-1}(t)\right)\right]^{\kappa-1}}{\left[\tau^{-1}(t)\right]^{\mathrm{K}-1} a\left(\tau^{-1}\left(\tau^{-1}(t)\right)\right)} \leq 1
$$

Assume that there exist positive functions $\vartheta, \zeta \in C^{1}\left(\left[t_{0}, \infty\right), \mathbb{R}\right)$ satisfying $\vartheta(t) \leq \delta(t)$, $\vartheta(t)<\tau(t), \zeta(t) \leq \delta(t), \zeta(t)<\tau(t), \zeta^{\prime}(t) \geq 0$ and

$$
\lim _{t \rightarrow \infty} \vartheta(t)=\lim _{t \rightarrow \infty} \zeta(t)=\infty
$$

If there exists a constant $\mu \in(0,1)$ such that the differential equations

$$
\psi^{\prime}(t)+\left(\frac{\left[\mu\left(\tau^{-1}(\vartheta(t))\right)\right]^{\kappa-1}}{(\kappa-1) !\left[b\left(\tau^{-1}(\vartheta(t))\right)\right]^{1 / \alpha}}\right)^{p-1} q(t)\left[a_{\kappa}(\delta(t))\right]^{p-1} \psi\left(\tau^{-1}(\vartheta(t))\right)=0
$$

and

$$
\phi^{\prime}(t)+\tau^{-1}(\zeta(t)) R_{\kappa-3}(t) \phi\left(\tau^{-1}(\zeta(t))\right)=0
$$

are oscillatory, then Eq. (1.1) is oscillatory.

Proof. Let $y$ be a non-oscillatory solution of (1.1) on $\left[t_{0}, \infty\right)$. Without loss of generality, we can assume that $y$ is eventually positive. It follows from Lemma 4 that there exist two possible cases (a) and (b).

Assume that the case (a) holds. From the definition of $w(t)$, we see that

$$
y(t)=\frac{1}{a\left(\tau^{-1}(t)\right)}\left[w\left(\tau^{-1}(t)\right)-y\left(\tau^{-1}(t)\right)\right] .
$$

By repeating the same process, we find that

$$
\begin{aligned}
y(t) & =\frac{w\left(\tau^{-1}(t)\right)}{a\left(\tau^{-1}(t)\right)}-\frac{1}{a\left(\tau^{-1}(t)\right)} \times\left\{\frac{w\left(\tau^{-1}\left(\tau^{-1}(t)\right)\right)}{a\left(\tau^{-1}\left(\tau^{-1}(t)\right)\right)}-\frac{y\left(\tau^{-1}\left(\tau^{-1}(t)\right)\right)}{a\left(\tau^{-1}\left(\tau^{-1}(t)\right)\right)}\right\} \\
& \geq \frac{w\left(\tau^{-1}(t)\right)}{a\left(\tau^{-1}(t)\right)}-\frac{1}{a\left(\tau^{-1}(t)\right)} \times \frac{w\left(\tau^{-1}\left(\tau^{-1}(t)\right)\right)}{a\left(\tau^{-1}\left(\tau^{-1}(t)\right)\right)} .
\end{aligned}
$$

Using Lemma 3, we get $w(t) \geq \varepsilon t w^{\prime}(t) /(\kappa-1)$ and hence the function $v^{1-\kappa}(t) w(t)$ is non-increasing which gives

$$
\left[\tau^{-1}(t)\right]^{\kappa-1} w\left(\tau^{-1}\left(\tau^{-1}(t)\right)\right) \leq \varepsilon\left[\tau^{-1}\left(\tau^{-1}(t)\right)\right]^{\kappa-1} w\left(\tau^{-1}(t)\right) .
$$

by the fact that $\tau(t) \leq t$.

Using (2.5), (2.4) turns out to

$$
y(t) \geq \frac{1}{a\left(\tau^{-1}(t)\right)}\left(1-\frac{\varepsilon\left[\tau^{-1}\left(\tau^{-1}(t)\right)\right]^{\kappa-1}}{\left[\tau^{-1}(t)\right]^{\mathrm{K}-1} a\left(\tau^{-1}\left(\tau^{-1}(t)\right)\right)}\right) w\left(\tau^{-1}(t)\right)
$$




$$
=a_{\mathrm{\kappa}}(t) w\left(\tau^{-1}(t)\right) .
$$

From (1.1) and (2.6), we obtain

$$
\left(b(t)\left[w^{(\kappa-1)}(t)\right]^{p-1}\right)^{\prime}+q(t)\left[a_{\kappa}(\boldsymbol{\delta}(t))\right]^{p-1}\left[w\left(\tau^{-1}(\boldsymbol{\delta}(t))\right)\right]^{p-1} \leq 0 .
$$

Since $\vartheta(t) \leq \delta(t)$ and $w^{\prime}(t)>0$, we get

$$
\left(b(t)\left[w^{(\kappa-1)}(t)\right]^{p-1}\right)^{\prime} \leq-q(t)\left[a_{\kappa}(\delta(t))\right]^{p-1}\left[w\left(\tau^{-1}(\vartheta(t))\right)\right]^{p-1} .
$$

Now, by using Lemma 1, we have

$$
w(t) \geq \frac{\mu}{(\kappa-1) !} t^{\kappa-1} w^{(\kappa-1)}(t) .
$$

for some $\mu \in(0,1)$. It follows from (2.7) and (2.8) that

$$
\begin{array}{r}
\left(b(t)\left[w^{(\kappa-1)}(t)\right]^{p-1}\right)^{\prime}+\left(\frac{\left[\mu\left(\tau^{-1}(\vartheta(t))\right)\right]^{\kappa-1}}{(\kappa-1) !}\right)^{p-1} q(t)\left[a_{\kappa}(\delta(t))\right]^{p-1} \\
\times\left[w^{(\kappa-1)}\left(\tau^{-1}(\vartheta(t))\right)\right]^{p-1} \leq 0
\end{array}
$$

for all $\mu \in(0,1)$.

Thus, if we set $\psi(t)=b(t)\left[w^{(\kappa-1)}(t)\right]^{p-1}$, then we see that $\psi$ is a positive solution of the first-order delay differential inequality

$\psi^{\prime}(t)+q(t)\left(\frac{\left[\mu\left(\tau^{-1}(\vartheta(t))\right)\right]^{\kappa-1}}{(\kappa-1) !\left[b\left(\tau^{-1}(\vartheta(t))\right)\right]^{1 /(p-1)}}\right)^{p-1}\left[a_{\kappa}(\delta(t))\right]^{p-1} \psi\left(\tau^{-1}(\vartheta(t))\right) \leq 0$.

It is well known (see [22, Theorem 1]) that corresponding Eq. (2.2) also has a positive solution, which is a contradiction.

Assume that the case (b) holds. Using Lemma 3, we get that

$$
w(t) \geq \varepsilon t w^{\prime}(t)
$$

and thus the function $w(t) / t$ is non-increasing, eventually. Since

$$
\tau^{-1}(t) \leq \tau^{-1}\left(\tau^{-1}(t)\right)
$$

we obtain

$$
\tau^{-1}(t) w\left(\tau^{-1}\left(\tau^{-1}(t)\right)\right) \leq \varepsilon \tau^{-1}\left(\tau^{-1}(t)\right) w\left(\tau^{-1}(t)\right) .
$$

Using (2.10), (2.4) turns out to

$$
\begin{aligned}
y(t) & \geq \frac{1}{a\left(\tau^{-1}(t)\right)}\left(1-\frac{\varepsilon \tau^{-1}\left(\tau^{-1}(t)\right)}{\tau^{-1}(t) a\left(\tau^{-1}\left(\tau^{-1}(t)\right)\right)}\right) w\left(\tau^{-1}(t)\right) \\
& =a_{2}(t) w\left(\tau^{-1}(t)\right),
\end{aligned}
$$


which yields with (1.1)

$$
\left(b(t)\left[w^{(\kappa-1)}(t)\right]^{p-1}\right)^{\prime}+q(t)\left[a_{2}(\delta(t))\right]^{p-1}\left[w\left(\tau^{-1}(\delta(t))\right)\right]^{p-1} \leq 0 .
$$

Since $\zeta(t) \leq \delta(t)$ and $w^{\prime}(t)>0$, we have that

$$
\left(b(t)\left[w^{(\kappa-1)}(t)\right]^{p-1}\right)^{\prime} \leq-q(t)\left[a_{2}(\delta(t))\right]^{p-1}\left[w\left(\tau^{-1}(\zeta(t))\right)\right]^{p-1} .
$$

Integrating (2.11) from $t$ to $\infty$, we obtain

$$
w^{(\kappa-1)}(t) \geq R_{0}(t) w\left(\tau^{-1}(\zeta(t))\right) .
$$

Now, integrating the above inequality from $t$ to $\infty, \kappa-3$ times, we obtain

$$
w^{\prime \prime}(t)+R_{\kappa-3}(t) w\left(\tau^{-1}(\zeta(t))\right) \leq 0 .
$$

Now, if we set $\phi(t):=w^{\prime}(t)$ and using (2.9), then we conclude that $\phi$ is a positive solution of the differential inequality

$$
\phi^{\prime}(t)+\tau^{-1}(\zeta(t)) R_{\kappa-3}(t) \phi\left(\tau^{-1}(\zeta(t))\right) \leq 0 .
$$

It is well known (see [22, Theorem 1]) that corresponding Eq. (2.3) also has a positive solution, which is a contradiction. The proof is complete.

Corollary 1. Assume that (2.1) holds and there exist positive functions $\vartheta$, $\zeta$ satisfying the conditions given in Theorem 1 . If

$$
\begin{array}{r}
\liminf _{t \rightarrow \infty} \int_{\tau^{-1}(\vartheta(t))}^{t}\left(\frac{\left[\tau^{-1}(\vartheta(s))\right]^{\kappa-1}}{\left[b\left(\tau^{-1}(\vartheta(s))\right)\right]^{1 /(p-1)}}\right)^{p-1} q(s)\left[a_{\kappa}(\delta(s))\right]^{p-1} \mathrm{~d} s \\
>\frac{1}{e}[(\kappa-1) !]^{p-1}
\end{array}
$$

and

$$
\liminf _{t \rightarrow \infty} \int_{\tau^{-1}(\zeta(t))}^{t} \tau^{-1}(\zeta(s)) R_{\kappa-3}(s) \mathrm{d} s>\frac{1}{e},
$$

then Eq. (1.1) is oscillatory.

Proof. It is well-known (see, e.g., [15, Theorem 2]) that Conditions (2.14) and (2.15) imply the oscillation of (2.2) and (2.3), respectively.

\section{EXAMPLES AND DISCUSSION}

We present two particular examples.

Example 1. Consider the equation

$$
\left[y(t)+a_{0} y(\tau t)\right]^{(\kappa)}+q_{0} t^{-\kappa} y(\lambda t)=0 ; \quad t \geq 1,
$$

where $q_{0}>0, \tau \in\left(a_{0}^{-1 /(\kappa-1)}, 1\right)$ and $\lambda \in(0, \tau)$. We note that $b(t)=1, a(t)=a_{0}$, $\tau(t)=\tau t, \delta(t)=\lambda t$ and $q(t)=q_{0} t^{-\kappa}$. Thus, if we choose $\vartheta(t)=\zeta(t)=\lambda t$, then 
it is straightforward to see that (2.1) and all the conditions given in Theorem 1 are satisfied. Moreover, we have

$$
\begin{aligned}
& a_{k}(t)=\frac{1}{a_{0}}\left(1-\frac{\tau^{1-k}}{a_{0}}\right) ; \quad k=2, \ldots, \kappa, \\
& R_{0}(t)=\frac{q_{0}}{(\kappa-1) a_{0}}\left(1-\frac{1}{\tau a_{0}}\right) t^{1-\kappa},
\end{aligned}
$$

and

$$
R_{\kappa-3}(t)=\frac{q_{0}}{(\kappa-1)(\kappa-2)(\kappa-3) ! a_{0}}\left(1-\frac{1}{\tau a_{0}}\right) t^{-2} .
$$

Hence, condition (2.14) and (2.15) become

$$
\frac{q_{0}}{a_{0}}\left(\frac{\lambda}{\tau}\right)^{\kappa-1}\left(1-\frac{\tau^{1-\kappa}}{a_{0}}\right) \ln \left(\frac{\tau}{\lambda}\right)>\frac{1}{e}(\kappa-1) !
$$

and

$$
\frac{\lambda q_{0}}{\tau a_{0}}\left(1-\frac{1}{\tau a_{0}}\right) \ln \left(\frac{\tau}{\lambda}\right)>\frac{1}{e}(\kappa-1) !
$$

respectively. It is clear to see that (3.2) implies (3.3). We end up with the resullt that (3.1) is oscillatory if (3.2) holds by Corollary 1.

Remark 1 . When $\kappa=4, a_{0}=16, \tau=1 / 2$ and $\lambda=1 / 3$ in Eq. (3.1), condition (3.2) yields $q_{0}>587.93$ which is better than that is obtained in [24], i.e., $q_{0}>4850.4$. Hence, our results improve those obtained in [24].

Example 2. Consider the particular equation

$$
[y(t)+(7 / 8) y(t / e)]^{(4)}+q_{0} t^{-4} y\left(t / e^{2}\right)=0 ; \quad t \geq 1,
$$

where $q_{0}>0$ is a constant, and

$$
p=2, \kappa=4, b(t)=1, a(t)=7 / 8, \tau(t)=t / \mathrm{e}, q(t)=q_{0} t^{-4}
$$

and $\delta(t)=t / \mathrm{e}^{2}$. If we apply the previous results to Eq. (3.4), then we get

(i) $q_{0}>113981.3$ by applying condition (1.4) in [25];

(ii) $q_{0}>3561.9$ by applying condition (1.5) in [27];

(iii) $q_{0}>3008.5$ by applying conditions (1.6)-(1.7) in [24].

Hence, the results of [24] improved those obtained in [25,27]. Furthermore, one can easily see that the criteria obtained in $[24,25,27]$ cannot be applied to (2.14) and (2.15) which demonstrates that our results are essentially new. 


\section{REFERENCES}

[1] R. P. Agarwal, S. R. Grace, and D. O'Regan, Oscillation theory for difference and functional differential equations. Kluwer Academic Publishers, Dordrecht, 2000. [Online]. Available: https://doi.org/10.1007/978-94-015-9401-1. doi: 10.1007/978-94-015-9401-1

[2] G. Aronsson and U. Janfalk, "On Hele-Shaw flow of power-law fluids," European J. Appl. Math., vol. 3, no. 4, pp. 343-366, 1992, doi: 10.1017/S0956792500000905. [Online]. Available: https://doi.org/10.1017/S0956792500000905

[3] B. Baculíková and J. Džurina, "Oscillation theorems for second-order nonlinear neutral differential equations," Comput. Math. Appl., vol. 62, no. 12, pp. 4472-4478, 2011, doi: 10.1016/j.camwa.2011.10.024. [Online]. Available: https://doi.org/10.1016/j.camwa.2011.10.024

[4] B. Baculíková and J. Džurina, "Oscillation theorems for higher order neutral differential equations," Appl. Math. Comput., vol. 219, no. 8, pp. 3769-3778, 2012, doi: 10.1016/j.amc.2012.10.006. [Online]. Available: https://doi.org/10.1016/j.amc.2012.10.006

[5] B. Baculíková, J. Džurina, and T. Li, "Oscillation results for even-order quasilinear neutral functional differential equations,” Electron. J. Differential Equations, pp. No. 143, 9, 2011.

[6] O. Bazighifan, E. M. Elabbasy, and O. Moaaz, "Oscillation of higher-order differential equations with distributed delay," J. Inequal. Appl., pp. Paper No. 55, 9, 2019, doi: 10.1186/s13660-019-2003-0. [Online]. Available: https://doi.org/10.1186/s13660-019-2003-0

[7] S. J. Bilchev, M. K. Grammatikopoulos, and I. P. Stavroulakis, "Oscillations of higher order neutral differential equations,” J. Austral. Math. Soc. Ser. A, vol. 52, no. 2, pp. 261-284, 1992.

[8] G. E. Chatzarakis, S. R. Grace, I. Jadlovská, T. Li, and E. Tunç, "Oscillation criteria for third-order emden-fowler differential equations with unbounded neutral coefficients," Complexity, vol. 2019, 2019, doi: 10.1155/2019/5691758. [Online]. Available: https://doi.org/10.1155/2019/5691758

[9] G. E. Chatzarakis, E. M. Elabbasy, and O. Bazighifan, "An oscillation criterion in 4thorder neutral differential equations with a continuously distributed delay," Adv. Difference Equ., pp. Paper No. 336, 9, 2019, doi: 10.1186/s13662-019-2281-3. [Online]. Available: https://doi.org/10.1186/s13662-019-2281-3

[10] M. K. Grammatikopoulos and I. P. Stavroulakis, "Necessary and sufficient conditions for oscillation of neutral equations with deviating arguments," J. London Math. Soc. (2), vol. 41, no. 2, pp. 244-260, 1990, doi: 10.1112/jlms/s2-41.2.244. [Online]. Available: https://doi.org/10.1112/jlms/s2-41.2.244

[11] M. K. Grammatikopoulos and I. P. Stavroulakis, "Oscillations of neutral differential equations," Rad. Mat., vol. 7, no. 1, pp. 47-71, 1991.

[12] I. Győri and G. Ladas, Oscillation theory of delay differential equations, ser. Oxford Mathematical Monographs. The Clarendon Press, Oxford University Press, New York, 1991, with applications, Oxford Science Publications.

[13] J. Hale, Theory of functional differential equations, 2nd ed. Springer-Verlag, New YorkHeidelberg, 1977, applied Mathematical Sciences, Vol. 3.

[14] I. T. Kiguradze and T. A. Chanturia, Asymptotic properties of solutions of nonautonomous ordinary differential equations, ser. Mathematics and its Applications (Soviet Series). Kluwer Academic Publishers Group, Dordrecht, 1993, vol. 89, translated from the 1985 Russian original. [Online]. Available: https://doi.org/10.1007/978-94-011-1808-8. doi: 10.1007/978-94-011-18088

[15] Y. Kitamura and T. Kusano, "Oscillation of first-order nonlinear differential equations with deviating arguments," Proc. Amer. Math. Soc., vol. 78, no. 1, pp. 64-68, 1980, doi: 10.2307/2043041. [Online]. Available: https://doi.org/10.2307/2043041 
[16] T. Li, B. Baculíková, J. Džurina, and C. Zhang, "Oscillation of fourth-order neutral differential equations with $p$-Laplacian like operators,” Bound. Value Probl., pp. 2014:56, 9, 2014, doi: 10.1186/1687-2770-2014-56. [Online]. Available: https://doi.org/10.1186/1687-2770-2014-56

[17] T. Li, Z. Han, P. Zhao, and S. Sun, "Oscillation of even-order neutral delay differential equations," Adv. Difference Equ., pp. Art. ID 184 180, 9, 2010, doi: 10.1186/1687-1847-2010-184180. [Online]. Available: https://doi.org/10.1186/1687-1847-2010-184180

[18] O. Moaaz, "New criteria for oscillation of nonlinear neutral differential equations," $A d v$. Difference Equ., pp. Paper No. 484, 11, 2019, doi: 10.1186/s13662-019-2418-4. [Online]. Available: https://doi.org/10.1186/s13662-019-2418-4

[19] O. Moaaz, E. M. Elabbasy, and O. Bazighifan, "On the asymptotic behavior of fourth-order functional differential equations," Adv. Difference Equ., pp. Paper No. 261, 13, 2017, doi: 10.1186/s13662-017-1312-1. [Online]. Available: https://doi.org/10.1186/s13662-017-1312-1

[20] O. Moaaz, E. M. Elabbasy, and A. Muhib, "Oscillation criteria for even-order neutral differential equations with distributed deviating arguments," Adv. Difference Equ., pp. Paper No. 297, 10, 2019, doi: 10.1186/s13662-019-2240-z. [Online]. Available: https: //doi.org/10.1186/s13662-019-2240-Z

[21] N. Parhi and A. K. Tripathy, "On oscillatory fourth order nonlinear neutral differential equations. I," Math. Slovaca, vol. 54, no. 4, pp. 389-410, 2004.

[22] C. G. Philos, "On the existence of nonoscillatory solutions tending to zero at $\infty$ for differential equations with positive delays," Arch. Math. (Basel), vol. 36, no. 2, pp. 168-178, 1981, doi: 10.1007/BF01223686. [Online]. Available: https://doi.org/10.1007/BF01223686

[23] C. Philos, "A new criterion for the oscillatory and asymptotic behavior of delay differential equations," Bull. Acad. Polon. Sci. Sér. Sci. Math., vol. 29, no. 7-8, pp. 367-370, 1981.

[24] G. Xing, T. Li, and C. Zhang, "Oscillation of higher-order quasi-linear neutral differential equations," Adv. Difference Equ., pp. 2011:45, 10, 2011, doi: 10.1186/1687-1847-2011-45. [Online]. Available: https://doi.org/10.1186/1687-1847-2011-45

[25] A. Zafer, "Oscillation criteria for even order neutral differential equations," Appl. Math. Lett., vol. 11, no. 3, pp. 21-25, 1998, doi: 10.1016/S0893-9659(98)00028-7.

[26] C. Zhang, R. P. Agarwal, and T. Li, "Oscillation and asymptotic behavior of higher-order delay differential equations with $p$-Laplacian like operators," J. Math. Anal. Appl., vol. 409, no. 2, pp. 1093-1106, 2014, doi: 10.1016/j.jmaa.2013.07.066. [Online]. Available: https://doi.org/10.1016/j.jmaa.2013.07.066

[27] Q. Zhang and J. Yan, "Oscillation behavior of even order neutral differential equations with variable coefficients," Appl. Math. Lett., vol. 19, no. 11, pp. 1202-1206, 2006, doi: 10.1016/j.aml.2006.01.003. [Online]. Available: https://doi.org/10.1016/j.aml.2006.01.003

\section{Authors' addresses}

O. Bazighifan

Hadhramout University, Department of Mathematics, Faculty of Science, Seiyun, Yemen

E-mail address: o.bazighifan@gmail.com

\section{S. R. Grace}

Cairo University, Department of Engineering Mathematics, Faculty of Engineering, 12221, Giza, Egypt

E-mail address: saidgrace@yahoo.com 


\section{J. Alzabut}

Prince Sultan University, Department of Mathematics and General Sciences, 11586, Riyadh, Saudi Arabia

E-mail address: jalzabutepsu.edu.sa

\section{A. Özbekler}

Atilim University, Department of Mathematics, 06830 Incek, Ankara, Turkey

E-mail address: aozbekler@gmail.com, abdullah.ozbekler@atilim.edu.tr 